\title{
NUTRIÇÃO CLÍNICA NA FACULDADE DE MEDICINA DE RIBEIRÃO PRETO - USP
}

\author{
CLINICAL NUTRITION IN THE FACULTY OF MEDICINE OF \\ RIBEIRÃO PRETO - UNIVERSITY OF SÃO PAULO
}

Helio Vannucchi ${ }^{1}$; José Ernesto dos Santos$^{2}$; Júlio Sérgio Marchini² \& José Eduardo Dutra de Oliveira ${ }^{1}$

${ }^{1}$ Professor Titular, ${ }^{2}$ Professor Associado da Divisão de Nutrologia do Departamento de Clínica Médica da Faculdade de Medicina de Ribeirão Preto da Universidade de São Paulo.

CorRESPONDÊNCIA: Helio Vannucchi - Divisão de Nutrologia do Departamento de Clínica Médica da Faculdade de Medicina de Ribeirão Preto da Universidade de São Paulo; CEP: 14048-900 - Ribeirão Preto - SP; Telefone (016) 602-3250; Email:hvannucc@fmrp.usp.br

VANNUCCHI H et al. Nutrição clínica na Faculdade de Medicina de Ribeirão Preto - USP. Medicina, Ribeirão Preto, 31: 7-12, jan./mar. 1998.

RESUMO: Os autores apresentam uma revisão a cerca do ensino de Nutrição, em Escolas Médicas, discutem questões filosóficas desse empreendimento médico, sua importância na formação do médico geral e suas perpectivas para o futuro, no Brasil e em todo o mundo. Apresentam as principais características do Curso de Nutrologia da Faculdade de Medicina de Ribeirão Preto - USP, enfatizam os objetivos, descrevem as atividades das quais participam os alunos do quarto ano médico, as aulas teóricas, práticas de enfermaria e ambulatório, específicas da Divisão de Nutrologia do Departamento de Clínica Médica. Discutem a demanda dos serviços de atendimento no Hospital das Clínicas da FMRP-USP, relacionando-os aos da divisão da Nutrologia.

UNITERMOS: Educação de Pré-Graduação em Medicina. Nutrição. Escolas Médicas. Hospitais Universitários.

\section{INTRODUÇÃO}

Os cuidados dispensados a pacientes hospitalizados tornaram-se extremamente complexos com o advento de modernas unidades de cuidados intensivos, melhorias no controle hemodinâmico e mecanismos de suporte de funções vitais. Não se deve, entretanto, ficar fascinado com o avanço da tecnologia e ignorar um dos itens mais importantes e elementares no cuidado desses pacientes - o suporte nutricional.

A desnutrição neutraliza as melhores intenções, mesmo aquelas consideradas exemplares. $\mathrm{O}$ estado nutricional do paciente influi decisivamente no resultado do tratamento da insuficiência respiratória, septice- mia, queimaduras sérias e traumatismo e, portanto, deve ser prioritariamente atendido, ao se tratarem pacientes em estado crítico. A resposta hipercatabólica à doença rapidamente leva o organismo à depleção em suas reservas energéticas e tecido muscular, especialmente se não for tratada, conduzindo a um risco ampliado de situações mórbidas. Um exemplo é o risco de infecção, o qual pode ser atenuado em certos pacientes por meio do suporte nutricional, como tem sido demonstrado de forma significativa ${ }^{(1,2)}$.

Os conhecimentos recentes fazem com que a desnutrição, observada em pacientes hospitalizados ou mesmo em portadores de doenças crônicas, seja inaceitável como um componente inexorável da situação. 
Em muitas ocasiões, a desnutrição ocorre porque a equipe médica, que atende o paciente, preocupa-se fundamentalmente com o tratamento de base, não dando qualquer importância à avaliação do estado nutricional ou à dietoterapia clínica. A alteração do estado nutricional é, sem dúvida, um dos graves problemas médicos. A grande incidência de desnutrição, observada em pacientes, na maioria dos hospitais, é considerada uma importante causa de maior morbidade e mortalidade intra-hospitalar ${ }^{(3)}$. Sabe-se que, muitas vezes, um paciente evolui para a morte devido não propriamente à doença de base, mas à desnutrição (que, muitas vezes, não aparece no diagnóstico médico) e à hospitalização prolongada, com o séquito indesejável de infecções adquiridas no hospital, alteração do nível de consciência, embolia pulmonar, arritmias cardíacas, etc. A desnutrição proteicocalórica, além de aumentar a taxa de morbidade e de mortalidade dos pacientes, está associada à diminuição da resposta ao tratamento clínico e/ou cirúrgico dispensados ${ }^{(4,5,6)}$.

A desnutrição é uma das condições mórbidas mais frequientes, atingindo de 25 a $70 \%$ dos pacientes hospitalizados, tanto em países desenvolvidos como naqueles em desenvolvimento. Cerca de $30 \%$ dos pacientes tornam-se desnutridos após a internação, e, além disso, a grande maioria dos pacientes desnutridos ao serem internados, piora no seu estado nutricional, após a hospitalização ${ }^{(7,8)}$. Em nosso meio, não existem dados objetivos sobre a prevalência de desnutrição intra-hospitalar, há mais de dez anos; no entanto, há sugestões de que, em alguns hospitais universitários, esteja ocorrendo uma queda discreta desta prevalência, fato que seria devido à criação de setores clínicos especialmente preocupados com a avaliação e suporte nutricional de pacientes hospitalizados. A desnutrição decorre dos efeitos catabólicos da doença subjacente e das restrições dietéticas impostas em função dos procedimentos diagnósticos e terapêuticos.

Historicamente, existe um atraso sócio-cultural quanto aos avanços da tecnologia. Em Medicina, não é incomum que tal demora atinja cinqüenta anos. Além disso, não é incomum que descobertas modificadoras iniciais levem décadas para se converterem em progressos aplicáveis. Os conhecimentos a respeito da necessidade de anti-sepsia estavam disponíveis cinqüenta anos antes que os médicos efetivamente iniciassem sua prática regular. Após o sucesso da anestesia geral, decorreram vinte e cinco anos para seu estabelecimento definitivo nos hospitais ${ }^{(9)}$. Em termos de suporte nutricional, são decorridos, agora, cerca de vinte cinco anos, desde a demonstração de sua impor- tância, enteral ou parenteral, e, hoje, o fornecimento de nutrição adequada é considerado uma obrigação médica, constituindo-se num direito básico do paciente.

Evolutivamente, pode-se constatar que só recentemente se encontram estudos e observações interessantes quanto ao uso de novas fórmulas, no tratamento dirigido às alterações de órgãos específicos (tratamento de pacientes com doenças renais crônicas - hepatopatas - insuficiência pulmonar ou situações de hipermetabolismo). "É possível que, no ano 2020, várias gerações de nutrientes específicos a cada doença tenham sido desenvolvidos, de tal forma que os pacientes poderão receber, diariamente, os nutrientes realmente necessários para sua situação específica" ${ }^{\text {(9). }}$.

Uma rápida consulta à 110 revistas médicas, no período de julho a dezembro (1994) mostra o seguinte panorama: foi possível encontrar 330 artigos relacionados à Nutrição, excluindo-se os periódicos específicos dessa subárea. Considerando-se as revistas clínicas, esse número foi de 259, (cerca de 43 artigos/mês). Ao considerar-se os periódicos eminentemente cirúrgicos, têm-se 71 artigos relativos à Nutrição, ou seja, 11,8 artigos/mês.

\section{ENSINO DE NUTROLOGIA NA FMRP}

O treinamento médico voltado para os problemas de ordem nutricional se tornam, então, imperativos na estrutura de ensino das Faculdades de Medicina. Entretanto, os dados disponíveis mostram que o ensino de Nutrição é inadequado ou inexistente na maioria das Escolas Médicas, no Brasil ou mesmo no exterior $^{(10 / 15)}$. Provavelmente, este problema está ligado ao pequeno número de profissionais ligados à área ou à inexistência de estrutura que possibilite ao médico privado subsistir profissionalmente longe dos hospitais universitários.

Em nosso meio, o curso de Nutrologia é ministrado para todos os alunos de Medicina, tendo por objetivo capacitá-los a diagnosticar diferencialmente as moléstias nutricionais, bem como conhecer o seu tratamento. Ensina e discute a fisiopatologia, o diagnóstico e o tratamento das moléstias nutricionais. $\mathrm{O}$ curso inclui a revisão de aspectos básicos de nutrição, discussões sobre dietoterapia e práticas de suporte nutricional enteral e parenteral. $\mathrm{O}$ ensino é centralizado no estudo de pacientes internados na enfermaria sob a responsabilidade da Divisão, no HC-FMRP, e dos pacientes atendidos no ambulatório. Inclui aulas teóricas, seminários, discussão de casos clínicos e visita médica aos leitos. 
Nos anos iniciais, o curso era destinado a alunos do sexto ano médico, incluindo discussões de casos clínicos e seminários sobre doenças nutricionais. No ano de 1975, o curso passou a ser ministrado aos alunos do quinto ano médico. A partir de 1978, o curso foi ministrado a grupos de dez a doze alunos do quarto ano médico, em rodízio pelo Departamento de Clínica Médica, com duração de cinco semanas, de fevereiro a dezembro, repetindo-se por oito vezes, durante o ano. Carga horária: atividades práticas - 45 horas; atividades teóricas - 30 horas. Atualmente, desde 1996, o curso foi subdividido em dois módulos. No primeiro, que dura uma semana, todos os alunos do terceiro ano recebem informações teóricas e práticas sobre a avaliação nutricional dos pacientes, o que inclui : anamnese, exame físico, exame antropométrico, bioimpedância e dados laboratoriais. No segundo módulo, 45 horas/turma, os alunos do quarto ano médico são divididos em turmas de dez a doze alunos, para efeito de discussão dos casos atendidos pela Divisão, tanto nas enfermarias quanto nos ambulatórios. Assistem a aulas teóricas, em grupos maiores, de trinta a trinta e cinco alunos, a respeito dos temas mais importantes de Nutrição Clínica, em nosso meio: desnutrição proteicocalórica, suporte nutricional via oral, enteral ou parenteral, alcoolismo e nutrição, obesidade, anorexia nervosa e bulimia, dislipidemias, deficiência de vitaminas, radicais livres, síndrome de má absorção, refratária a dietoterapia, via oral, síndrome do intestino curto.

De modo geral, tem-se conseguido transmitir a importância dos benefícios reais que são oferecidos aos pacientes, ao se promover a melhoria do estado nutricional. A Nutrologia é um novo campo de aplicação médica e deve contar, para seu pleno desempenho, com a participação de outros profissionais com atividades correlatas (nutricionista, farmacêutico bioquímico e enfermeira). Dentro de uma Faculdade de Medicina, em uma estrutura de ensino médico, a Nutrologia só terá futuro e boas perspectivas, se estiver integrada junto aos Departamentos de Clínica Médica, a despeito de sua inegável importância em Saúde Pública. ANutrologia está se consolidando, rapidamente, como especialidade clínica, respondendo à necessidade de melhor compreender e utilizar conhecimentos de alimentação e nutrição nos processos metabólicos normais e patológicos, modificados por falta ou excesso de nutrientes ou por alterações metabólicas, desencadeadas por doenças não nutricionais ${ }^{(16 / 20)}$.

Outra atividade, considerada importante para a concretização da atividade de Clínica Nutricional e reconhecimento da especialidade médica, se refere ao treinamento de médicos residentes. Dessa forma, têm-se desenvolvido atividades de ensino de residentes de primeiro, segundo e terceiro anos, orientando-os em problemas de enfermaria e ambulatório, relacionados à Nutrologia, desde 1979.

Os principais fatores de sustentação para o empreendimento - Nutrição Clínica - têm sido, até agora, entre nós, a proximidade de um centro universitário de excelência na área, laboratórios especializados e o bom aproveitamento dos contatos, no exterior, com grupos equivalentes ${ }^{(21 / 26)}$.

Cabe, então, aos grupos de Nutrição Clínica desenvolver condições de trabalho no sentido de permitir o tratamento global de pacientes, diminuindo, assim, a morbidade observada atualmente.

Nos próximos anos, resolvidos os problemas técnicos e éticos, o direcionamento da pesquisa para o melhor conhecimento da biologia molecular, poderá eventualmente oferecer alternativas clínicas valiosas, especialmente quanto a certas doenças degenerativas, decorrentes de erros alimentares crônicos.

O médico especialista em nutrição - nutrólogo - deve ter treinamento específico, com conhecimentos aprofundados da fisiopatologia, diagnóstico e tratamento (medicamentoso e dietético) dos pacientes com problemas relacionados aos nutrientes e energia ${ }^{(27,28)}$.

Em relação ao atendimento dos pacientes atendidos pela Divisão de Nutrologia, no HCFMRP-USP, é possível distinguir três subáreas de interesse. Uma delas se refere ao tratamento de pacientes com deficiências nutricionais, alcoolismo, problemas nutricionais relacionados às doenças hepáticas e pancreáticas. Outra, se aplica ao atendimento de pacientes com doenças relacionadas à ingestão anormal de alimentos, como anorexia nervosa, bulimia e obesidade, assim como as alterações do metabolismo de lípides. A terceira área se refere ao tratamento nutricional de pacientes com síndromes de má absorção intestinal.

Como mostrado na Figura 1, durante os últimos anos, foram atendidos, nos ambulatórios da Divisão, cerca de um mil pacientes/ano, com problemas nutricionais, número comparável ao de outras divisões do Departamento de Clínica Médica. Quando se considera o número de pacientes internados nas enfermarias de Nutrologia do HC-FMRP, este se compara ao de outros departamentos ou outras especialidades do HC - Figura 2.

Ressalta-se que a Nutrologia utiliza técnicas próprias e equipamentos para o estabelecimento do 


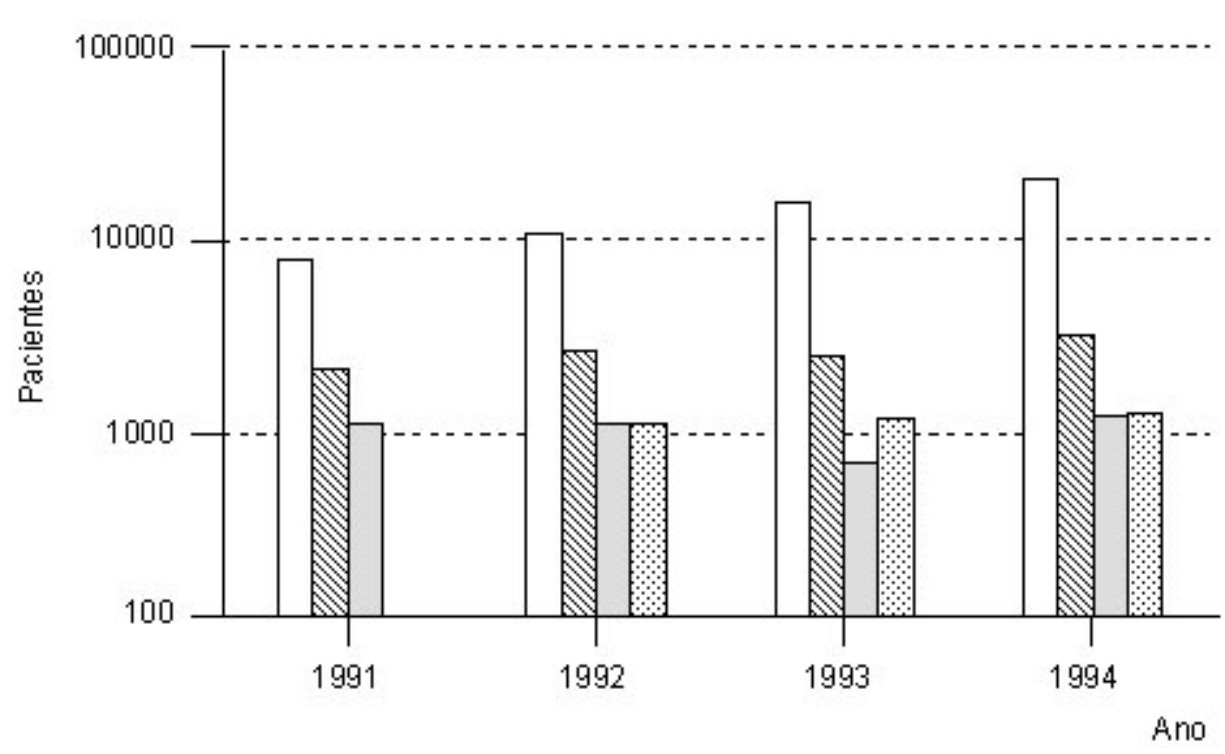

Cardiologia $\mathbb{N}$ Nefrologia

Nutrologia $\quad$ Geriatria

Figura 1 - Número de pacientes ambulatoriais, seguidos por diferentes divisões clínicas do Departamento de Clínica Médica da Faculdade de Medicina de Ribeirão Preto.

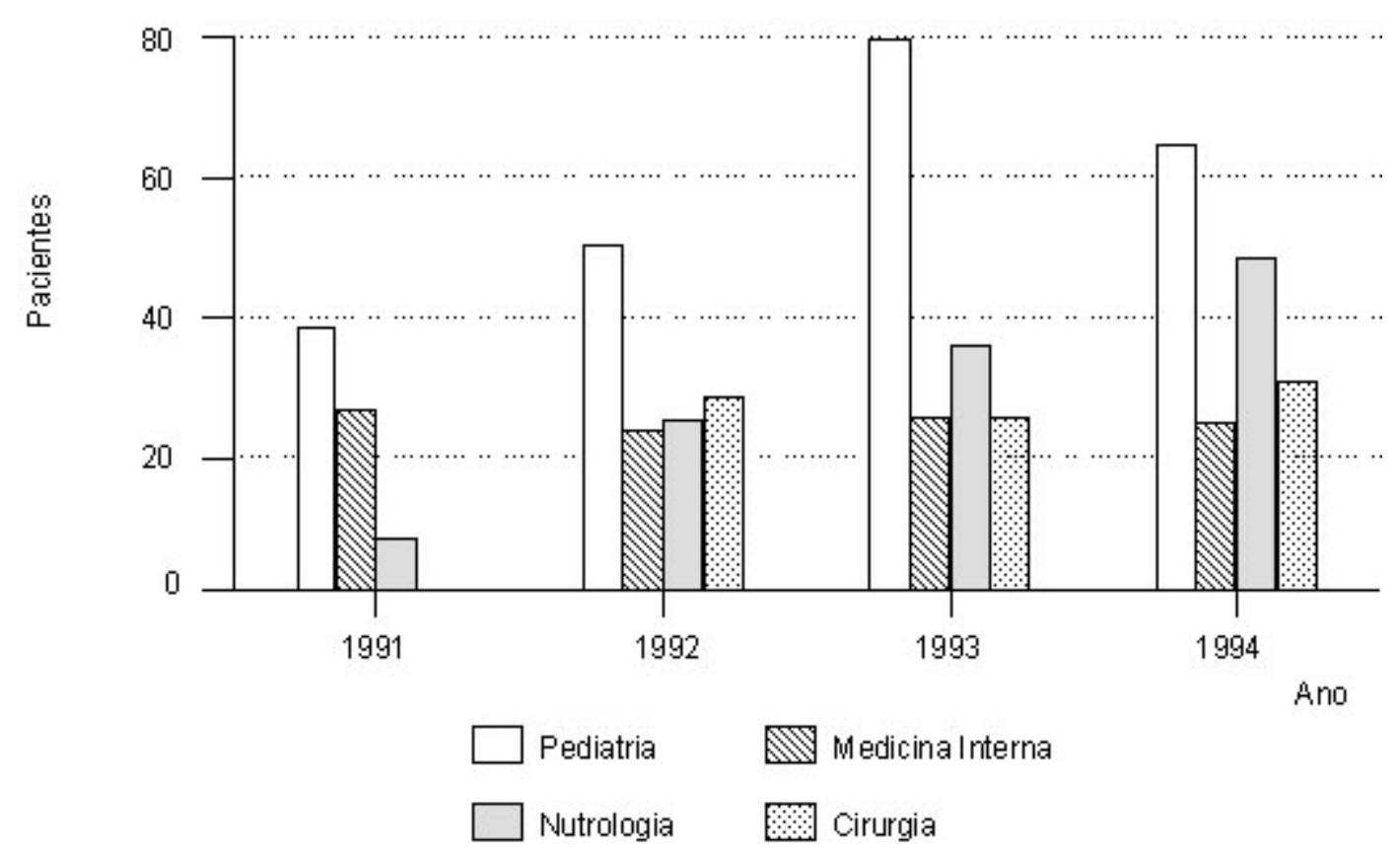


diagnóstico ou avaliação da ingestão alimentar ou ainda para conhecer a composição corporal dos pacientes. Além disso, conta com técnicas específicas de laboratório clínico, como determinação dos níveis orgânicos de vitaminas e minerais e ainda novas técnicas de isótopos estáveis e biologia molecular para o melhor entendimento de vias metabólicas e o estado nutricional em situações de doença (Tabela I).
Havendo disponibilidade para o treinamento clínico em Nutrição, uma infra-estrutura adequada deve existir no ambiente hospitalar, para o estudo dos problemas nutricionais e, se essa infra-estrutura (incluindo a equipe médica e paramédica, programas de ensino, projetos de pesquisa e técnicas peculiares de avaliação do estado nutricional de pacientes) é possível, a identidade da nutrição clínica estará garantida.

Tabela I - Procedimentos utilizados no acompanhamento nutricional de pacientes em geral

\begin{tabular}{|c|c|c|}
\hline & Procedimento & Objetivo \\
\hline Primeira visita & História e exame físico feito pelo médico & Diagnóstico clínico e detecção \\
\hline $\begin{array}{l}\text { Avaliação da ingestão } \\
\text { de nutrientes }\end{array}$ & Método recordatório & $\begin{array}{l}\text { Correlação entre doença e nutrien- } \\
\text { tes específicos }\end{array}$ \\
\hline Antropometria & $\begin{array}{l}\text { Peso, altura, prega tricipital } \\
\text { Índice de massa corporal* } \\
\text { Reserva gorda do braço** }\end{array}$ & Alterações da massa corporal \\
\hline Composição corporal & Bioimpedância, tomografia computadorizada & Diagnóstico e seguimento \\
\hline Dados bioquímicos & $\begin{array}{l}\text { Nutrientes no plasma e urina, testes de es- } \\
\text { tímulo e sobrecarga. }\end{array}$ & Diagnóstico e seguimento \\
\hline Outras técnicas & $\begin{array}{l}\text { Ressonância nuclear magnética, isótopos } \\
\text { estáveis, biologia molecular }\end{array}$ & $\begin{array}{l}\text { Estudo do metabolismo intermediário } \\
\text { e celular }\end{array}$ \\
\hline
\end{tabular}

${ }^{*}$ Peso/ altura $\left(\mathrm{kg} / \mathrm{m}^{2}\right)$

** Prega tricipital/ comprimento do braço $\left(\mathrm{mm} / \mathrm{dm}^{2}\right)$

VANNUCCHI H et al. Clinical nutrition in the Faculty of Medicine of Ribeirão Preto - University of São Paulo. Medicina, Ribeirão Preto, 31: 7-12, jan./march 1998.

ABSTRACT: The efficiency of nutrition support division in patient care is linked to the status of clinical nutrition in teaching hospitals. There should be opportunities for physicians interested in Clinical Nutrition to be trained and have a profile to other clinical specialists. The Clinical Nutrition Group in the Department of Internal Medicine at the teaching hospital of the Medical School of Ribeirão Preto, Brazil, is an example of a functioning clinical unit. Statistics are presented comparing patients number for the Clinical Nutrition Group at Ribeirão Preto with other clinical services (Cardiology, Nephrology and Geriatrics). Clinical Nutrition has its own clinical methodology and technology. When Clinical Nutrition will extend beyond hospital boundaries and become useful in the primary care of patients at the community level.

UNITERMS: Education, Medical, Undergraduate. Nutrition. Schools, Medical. Hospitals, University

\section{REFERÊNCIAS BIBLIOGRÁFICAS}

1 - MOORE FA et al. TEM versus TPN following major abdominal trauma-reduced septic morbidity. J Trauma 29: 916-922, 1989.

2 - KUDSD KA et al. Enteral versus parenteral feeding. Effects on septic morbidity after blunt and penetrating abdominal trauma. Am Surg 215: 503-511, 1992.
3 - VANNUCCHI H; CUNHA DF \& BERNARDES MM. Avaliação dos níveis séricos das vitaminas $A, E, C$ e $B_{2}$, de carotenóides e zinco, em idosos hospitalizados. Rev Saúde Pública 28: 121-126, 1994.

4 - PIZOTTI NJ; FAINTUCH J \& ROCHAA. Influence of clinical and nutritional factors on the prognosis of critically ill patients-the role of enteral nutrition. Rev Hosp Clin Fac Med São Paulo 42: 159-163, 1987. 
5 - DEMPSEY DT; MULLEN JL \& BUZBY GP. The link between nutritional status and clinical outcome: can nutritional intervention modify it? Am J Clin Nutr 47: 352-356, 1988.

6 - COATS KG et al. Hospital-associated malnutrition: a reeavaluation 12 years later. J Am Diet Assoc 93: 27-33, 1993.

7 - BISTRIAN BR et al. Protein status of general surgical patients. JAMA 230: 858-860, 1974.

8 - BLACKBURN GL et al. Nutritional and metabolic assessment of the hospitalized patient. JPEN J Parenter Enteral Nutr 1: 11-22, 1977.

9 - SELTZER MM. Education and training strategies for improving the quality of care. Nutr Int 3: 35-36, 1987.

10 - VANNUCCHI H et al. Educação médica - Ensino de nutrologia na Faculdade de Medicina de Ribeirão Preto - Sua evolução e objetivos. Medicina, Ribeirão Preto 10: 31-34, 1979.

11 - DUTRA DE OLIVEIRA JE. Teaching Nutrition in Medical Schools: Past, Present and Future. World Rev Nutr Diet 25: 142-163, 1976.

12 - BISTRIAN BR. Thoughts on training and employment opportunities in clinical nutrition. Am J Clin Nutr 49: 180-182, 1989.

13 - MCLAREN DS. Nutrition in Medical Schools: a case of mistaken identity. Am J Clin Nutr 59: 960-963, 1994.

14 - SOUZA N; VANNUCCHI H \& DUTRA DE OLIVEIRA JE. The teaching of nutrition in Brazilian Medical Schools. World Rev Nutr Diet 28: 215-217, 1981.

15 - DUTRA DE OLIVEIRA JE; VANNUCCHI H \& VENEZIANO PR. L'enseignement de la nutrition dans le facultës de medicine au Brésil. Une experience pratique. Med et Nutr (Paris) 15: 413- 416, 1979.

16 - WAHLQVIST ML \& ISAKSSON B. Training in clinical nutrition: undergraduate and postgraduate. Lancet 2: 1295-1297, 1983.

17 - WEINSER RL. Nutrition education in U.S. Medical Schools: A Status Report 1985. Nutr Int 1: 30-36, 1985.
18 - ZIMMWERMANN M \& KRETCHEMER N. Isn't time to teach nutrition to medical students? Am J Clin Nutr 58: 828-829, 1993.

19 - HALSTED CH. Certification of clinical nutricionists. Am J Clin Nutr 62: 10-12, 1995.

20 - HODGES RE. Nutrition education in the clinical years. Am J Clin Nutr 30: 803-805, 1977.

21 - DUTRA DE OLIVEIRA JE. Teaching nutrition in Medical Schools: some Problems and proposed solutions. J Nutr Educ 6: 49-51, 1974

22 - DUTRA DE OLIVEIRA JE \& MARCHINI JS. Clinical nutrition for MDs: reapraisal and identity. Am J Clin Nutr 62: 1289-1290, 1995.

23 - YOUNG EA. National Dairy Council Award for excellence in Medical/Dental Nutrition Education Lecture, 1992: Perpectives on nutrition in medical education. Am J Clin Nutr 56: 745-751, 1992.

24 - BERMAN EL. President adress, 1992: The American Society for Clinical Nutrition-a glance backward, a look ahead. Am J Clin Nutr 56: 726-729, 1992.

25 - DUTRA DE OLIVEIRA JE; VANNUCCHI H \& DOS SANTOS JE. Clinical nutrition:where do we stand? A view from Brazil. Hum Nutr Clin Nutr 6: 116-119, 1984.

26 - VANNUCCHI H \& DUTRA DE OLIVEIRA JE. Nutro-logiaNutrição Clínica - Arq Bras Med 59: 83-86, 1985.

27 - DUTRA DE OLIVEIRA JE. Views on Nutrition Programs in Brazil. Am J Clin Nutr 11: 172-173, 1962.

28 - DUTRA DE OLIVEIRA JE \& MARCHINI JS. Primary care physicians and clinical nutrition: can good medical nutrition care be offered without well-trained physicians in the area?. Am J Clin Nutr 65: 2010S - 2012S, 1997.

Recebido para publicação em: 30/01/98

Aprovado para publicação em: 25/02/98 